\title{
TELEMEDICINE IN ASSISTANCE TO HEALTHCARE IN THE COVID-19 PANDEMIC
}

\author{
K. Kilova ${ }^{1}$, S. Uzunova² \\ ${ }^{1}$ Department of Medical Informatics, Biostatistics and e-Learning, Faculty of Public Health, \\ Medical University of Plovdiv - Plovdiv, Bulgaria \\ ${ }^{2}$ Faculty of Public Health, Medical University of Plovdiv - Plovdiv, Bulgaria
}

\begin{abstract}
Global pandemics pose unprecedented challenges to healthcare. In the current situation, telemedicine appears to be a sustainable and effective solution in the implementation of precautions, prevention and treatment in order to discontinue the spreading of the coronavirus infection (COVID-19). The aim of this paper was to research and analyze the role of telemedicine in assistance to healthcare in the COVID-19 pandemic. Methods: The PubMed Database, Google Scholar, SpringerLink, ScienceDirect (Elsevier) and sites of public authorities have been accessed and reviewed. Results: Advantages, restrictions, policy and application of telemedicine in the conditions of COVID-19 pandemic have been studied and analyzed. Conclusion: Healthcare systems are put under an extreme test during global pandemics. Telemedicine is crucial during the COVID-19 pandemic because it guarantees that patients have access to medical care.
\end{abstract}

Key words: telemedicine, COVID-19 pandemic, health, healthcare

Corresponding author: Kristina Kilova, Department of Medical Informatics, Biostatistics and e-Learning, Faculty of Public Health, Medical University of Plovdiv, Bulgaria, 15A "Vasil Aprilov" Blvd., 4002 Plovdiv, Bulgaria, e-mail:k_kilova@yahoo.com

\section{INTRODUCTION}

$\mathrm{O}$ n February 12, 2020 the new coronavirus was named severe acute respiratory syndrome Coronavirus 2 (SARS-CoV-2), while the associated disease was called COVID-19.

On January 30, 2020 the World Health Organization (WHO) identified the incidence of coronavirus infection (COVID-19) as a global health emergency and on March 11, 2020 its Director - General Tedros Adhanom Gebreysus described COVID-19 as a pandemic [1].

COVID-19 poses a risk to the public health via the international spread of the disease and requires coordinated international response. Since its appearance whole countries have been quarantined, millions of people have been living in extreme conditions, and over 400,000 have deceased (https://www.worldometers.info/coronavirus/). This without any doubt puts the healthcare systems under an extreme test. Telehealth services, also known as remote or virtual care, can help triage patients and retain them in their homes away from congested hospitals and clinics. The positive contribution of telemedicine during the pandemic is indisputable, but these technologies also have certain limitations.

The purpose of this review was to explore and discuss the role of telemedicine in assistance to healthcare in the COVID-19 pandemic. 


\section{METHODS}

The PubMed Database, Google Scholar, SpringerLink, ScienceDirect (Elsevier) and sites of public authorities have been accessed and reviewed.

\section{RESULTS}

\section{Advantages in the conditions of COVID-19}

During the current global pandemic, telehealth is emerging as an effective and sustainable solution acting as precautions, prevention and treatment to discontinue the dissemination of COVID-19. The benefits are numerous - both to patients and healthcare professionals as well as to public health in general. Patients can access vital health services via video conferencing, remote monitoring, electronic counseling and wireless communications while receiving sufficient care without leaving their home. This could reduce their exposure to the risk of contamination with the disease. Telemedicine can help communities in remote or rural areas with few health services and limited staff because it overcomes the barriers of distance and time between healthcare providers and patients. Moreover, telehealth could overcome the gap among people, medical professionals and healthcare systems, allowing patients, especially symptomatic ones, to stay home and communicate with their doctor through virtual channels, helping to reduce the spread of the coronavirus. Furthermore, using telehealth solutions for immediate communication is a way to reduce the pressure on emergency rooms and clinics by limiting contact and, thus, the dissemination of the virus. Telemedicine provides a 24/7 lifeline for patients to contact medical service providers. Patients with mild acute respiratory infection can be monitored closely and daily and if there are any changes in their clinical condition, appropriate actions can be taken immediately [2]. Of note, telemedicine solutions provide not only care but also psychological support for patients who are afraid of the potential symptoms associated with the new coronavirus.

Another advantage of telemedicine is that the access to consultations with specialists who share COVID-19 experience is expanding. A medical practice or hospital system may immediately consult more qualified or missing medical professionals. This makes it easier for physicians working at a remote practice to seek different opinion about their patient and for patients to see a specialist, regardless of their location [3].

Telemedicine tools and technologies can assist the epidemiological surveillance by helping to identify new cases and illustrate trends [4]. It is also pos- sible to monitor the development of the disease and improve communication to plan and mobilize emergency teams [5].

Describing the many advantages of telemedicine solutions in a pandemic, the focus is on a few of them that would play a major role:

1. Patients could be examined remotely instead of attending a medical practice or hospital. The possibility of remote medical triage is created by separating patients with common cold and flu-like symptoms from those who do not need medical intervention or can receive it at home. With this solution, potentially healthy people are out of the hospital or doctor's office, alleviating the health system and reducing the risk of transmitting the virus.

2. Telemedicine can help provide routine care to patients with chronic diseases who are at risk because the virus could be very harmful and even fatal to them.

3. Medical professionals are at an increased risk of becoming infected with COVID-19 due to their permanent contact with a large number of patients. Once tested and confirmed, they will be quarantined and left out of the healthcare system just when it needs them most. However, in the presence of telehealth technology during quarantine, these specialists are able to continue to examine their patients via remote access.

One of the biggest advantages of telemedicine in pandemic conditions is the ability to discontinue the dissemination of the virus by symptom-free patients. Leaving their home, they could spread the disease at the medical practice or at the entire emergency department, putting everyone at risk. At the same time, patients have access to medical professionals and reliable health information, avoiding hospitals or public transportation.

By implementing modern telehealth solutions, doctors can expand their reach, and even if they are quarantined, they could remotely examine and diagnose more patients in a shorter period of time, reducing the number of patients entering hospitals. This is an opportunity to actually reduce the burden on the health systems.

\section{Restrictions in the conditions of COVID-19}

One of the most significant limitations to the use of telemedicine in the context of COVID-19 is that most hospitals and major medical practices are not adequately equipped to provide care in this way. Health managers are just beginning to understand that telemedicine technologies are the key solution to retain potentially infected people outside hospitals and doc- 
tor's offices. As this public health crisis continues to escalate, telemedicine rapidly gains recognition as a critical tool for slowing the spread of COVID-19. Unfortunately, it cannot help some patients who are in a more serious condition.

A number of inconveniences are created for medical practices. On the one hand, managers must establish a system for informing patients that office visits can be replaced by telemedicine consultations, and, on the other hand, they have to train specialists on offering these services, reviewing planning processes, determining triage procedures, setting up communication systems and forming charging practices. This is not necessarily a long process, but it is useful for providers to have a set of guidelines to facilitate the transition.

Another limitation is the lack of hardware. While many telemedicine programs are a matter of software solution, medical service providers must also have the adequate equipment for the type of examination - camera quality, sound, internet connection, etc. The better the technology, the better the patient consultation.

Some of the main obstacles to the faster implementation of these services are insufficient training and education of consumers. Medical professionals need to be trained on the new healthcare method. Patients, on the other hand, should be informed about the purpose of telecare and should be instructed how to safely and securely use the devices [6]. The lack of sufficient computer-literate professionals with experience in managing computer services, combined with the lengthy process required to master computerbased medical devices, could hinder the implementation of telehealth services [7].

Telecare is inapplicable for many medical specialties and seriously ill patients, especially in the absence of peripheral devices that register their health indicators.

\section{Policy change}

Historically, healthcare providers who want to proffer clinical services through telemedicine face a number of legal and regulatory challenges. These include issues such as licensing, jurisdiction, reimbursement of services and ensuring compliance with legal regulations and medical standards. In recent months, national and international regulations have changed significantly to encourage greater uptake and use of telemedicine and other digital health technologies for the provision of clinical services [8].

This year both federal and national laws have been amended in the United States to facilitate the wider use of telemedicine platforms. Legal and regulatory changes include expanding of telemedicine services, reducing technological requirements, implementing new licensing and lending approaches, and easing supervisory laws related to providers. Congress passed an emergency spending bill providing USD 8.3 billion to combat COVID-19, including USD 500 million for telemedicine services. And while most stock exchanges are disappearing, shares of virtual health companies are rising [9].

In light of the public health emergency, the US Department of Health Civil Rights Office (HHS) has announced that it will not impose sanctions on physicians who use telehealth in the event of non-compliance with regulatory requirements under the Health Insurance Portability and Accountability Act (HIPAA) as long as the confidentiality of personal data is respected. Allowed platforms that are not HIPAA compliant include Apple FaceTime, video chat on Facebook Messenger, video on Google Hangouts, video chat on WhatsApp or Skype [10].

Several countries have also temporarily waived restrictions on doctor licensing in order to allow greater use of telemedicine services during this public health crisis [11]. The international legal and regulatory environment has also witnessed a significant change in the adaptation of telemedicine technologies in recent months. Many countries in Asia have amended their laws to allow greater adoption of telemedicine systems, provided that procedures are in place for digital health technologies and cyber security.

In Austria, remote diagnosis, remote treatment or remote consultation are prohibited under the Medical Act, but are included in the current program of the Austrian Federal Government. Electronic prescriptions are legally permissible, while all medication prescriptions must be digitized by May 2022 .

In Poland the Medical Activity Act allows remote health services from 2015 and patients can seek advice and get a diagnosis without having to visit a doctor's office. Telemedicine is used to diagnose and monitor the health status of patients with chronic conditions such as asthma, cardiovascular disease, diabetes, mental illnesses and stroke. It is mainly intended for patients who are not mobile or live in rural areas where access to healthcare is difficult. According to an amendment to the Medicines Act in Poland, prescriptions can be issued electronically by sending them to patients by e-mail, SMS or as a printout [12].

In Romania the law generally requires that any medical decision has to be made through a personal examination of the patient by a relevant doctor. Remote examination or medical intervention is only permitted 
if it aims to help doctors give a diagnosis, establish adequate treatment, or take another specialized measure to finalize the required actions. Restrictions imposed by Romanian authorities during the coronavirus pandemic kept many people at home. In this context, many public and private hospitals began offering telemedicine services in mid-April. Private health operators, Regina Maria, in partnership with Microsoft Romania, have launched the Virtual Clinic platform for free medical advice. MedLife has launched a video conferencing service that offers online help for a number of specialties, with more than 80 doctors included. The services are available completely free of charge. The Doclandia telemedicine platform offers its services free of charge throughout the state of emergency, with the possibility to involve all doctors in Romania [13].

In Bulgaria there is no explicit regulation or legislation according to which telemedicine services can be provided, and there is no legal definition of the terms. Nevertheless, telemedicine is included in the medical standard in the specialty of Emergency Medicine, as well as in the ordinance on rare diseases [14, 15].

At the same time, there is a supply of telemedicine services from various applications, as well as from independent medical practices and health insurance funds, and most of them are also offered free of charge to consumers.

\section{Applications}

In the conditions of a pandemic, via the application of ICT and online resources on the internet, we have access to a huge amount of information.

\section{Access to information}

Numerous platforms and websites have been created, providing guidance and constantly updated information - both for patients and medical professionals. A wave of information is flooding into us from all sides - on a regional, national and international level. From home isolation and reduced access to qualified care, the presence of incorrectly presented and misleading information can lead to deterioration of certain groups of patients, mental changes and in many cases to serious consequences on individuals themselves, their family and society as a whole.

It is extremely important that the information provided is adequate and does not cause panic among the population. An example in this regard is the Internet platform Digital health, where up-to-date data can be found in relation to COVID-19 - for both patients and medical professionals [16].

\section{Innovation}

In Israel, Tyto Care's telehealth solutions are integrated to examine patients with COVID-19 in quarantine wards, as well as to monitor patients in isolation at home by supplying the TytoHome kit. Tyto Care fully realizes the potential of telehealth at this critical time. This is a hand-held modular medical kit for examining the heart, lungs, stomach, skin, throat, ears and body temperature, including unique technology for orientation, navigation and quality analysis in full compliance with relevant regulatory approvals, including Health Canada, HIPAA (Health Insurance Portability and Accountability Act) and GDPR (EU Data Protection Act). This allows physicians to obtain the vital clinical data they need to monitor the situation from a safe distance, minimizing physical contact and reducing the heavy workload of clinics. The solution can be implemented quickly and on a large scale, as well as the training of medical staff and its implementation are possible within one day. The medical kit is delivered to the patient's home together with easy instructions for use [17].

\section{Patient care}

It is very important to encourage citizens to have access to health portals to find accurate health information. In a short time, websites and mobile applications have been created on the internet to inform patients about the coronavirus infection, ways to prevent it, hygiene rules, and proximity to a doctor or hospital. Artificial intelligence is integrated into online-based algorithms that allow anyone to quickly test for symptoms of the coronavirus infection (https://oncare.org/).

According to a recent survey of American patients, $84 \%$ are more likely to choose a provider that offers telemedicine over one that does not, so it is clear that this technology is something that patients demand. Today, practitioners are investing in telemedicine in view of the current pandemic, but they must also see the issue as a long-term investment to provide better services to patients [18].

\section{Mobile apps}

Given the global situation in which the world finds itself today, many governments, companies and civic movements have developed various initiatives to inform the population and help manage the crisis. On the website of the European Center for Mobile Health a preliminary and non-exhaustive list can be found, compiled with great effort in a short time. A number of mobile phone applications have been developed and continue to complement each other, including opportunities for video consultations in different lan- 
guages, advice, finding a testing center or a doctor nearby and many other options offering solutions and help [19].

In Japan, the Ministry of Health, Labor and Welfare provided 2000 mobile phones to passengers and crew who were in isolation on the Diamond Princess cruise ship to allow a teleconsultation with a doctor. In South Korea, the government's strategy to control the spread of disease is supported by a mobile application that directs people to go through test centers and monitors the movement of those people who are tested positive [20].

Apple's Siri virtual assistant has been updated to provide symptom-based guidance and links to COVID-19 websites. If the user asks Siri "How do I know if I have coronavirus?" or "I think I have coronavirus", the tool initiates a question to determine the user's current symptoms. If the user tells Siri that the symptoms are severe but not life-threatening, the virtual assistant advises him to call his doctor and provides a selection of telemedicine companies that include Amwell, Teladoc, Doctor on Demand, HealthTap, PlushCare, Sydney Care and MDLive [21].

Guides for implementation of telemedicine services in practices

The American Medical Association has created a new resource for physicians to integrate telehealth services in the age of the coronavirus. The guide is aimed at assisting physicians in applying the technology. The guidelines cover everything from policy and coding to implementation. The association also launched a virtual discussion on telemedicine and COVID-19, where clinicians can contribute and share experiences involving doctors considered experts. "Telemedicine and technology are crucial ways to provide care and preserve our healthcare professionals, patients and vulnerable populations, especially in the wake of the COVID-19 pandemic," the panel said. "This discussion is intended to share best practices and the latest policy and payment updates in support of physicians and practices to accelerate the application of telemedicine." [22].

A detailed guidebook to everything medical professionals need to know about offering telecare in a pandemic is published on the pages of various professional organizations in the United States: the American Academy of Physicians, the American Academy of Ophthalmology, Cardiology, and many others [23]. Most telemedicine platforms also offer training modules to help users launch their software so that practitioners can provide services quickly.
In search of a solution

The main focus of the response efforts of governments and health organizations worldwide is to overcome the crisis and stop the spread of COVID-19. But the current urgent public emergency requires a system to further prevent the depletion of medical supplies and resources.

Telemedicine systems are ideal for alleviating the overcrowding of hospitals and clinics, while preventing further unnecessary human exposure and promoting the provision of high quality care. As state, federal, and international laws expand in recent months and weeks to achieve greater acceptance of telemedicine systems, health care providers are considering and implementing such systems. Although some legal and regulatory challenges remain, the outbreak of COVID-19 may prompt legislators and regulatory agencies to promulgate additional measures that facilitate the adoption of telemedicine.

A joint project of Apple and Google has been launched, which will be presented in May. With special software on iPhone and Android phones, it will be possible to track the spread of coronavirus, and users will be notified if they have been in contact with an infected person and are potentially ill. The new technology will rely on Bluetooth, commonly used for short-distance communication between devices. The software will consist of an API (application programming interface) that allows phones with different operating systems to exchange data with each other. A similar application is used in Singapore - TraceTogether, and is adopted by $12 \%$ of the population [24].

In early March 2020, the European Commission announced that it was looking for start-ups that could provide innovative solutions for testing, monitoring and treating COVID-19. In this regard, the Bulgarian Professional Association of Robotics and Automation has published a list of solutions related to coronavirus that fall into the field of technologies and innovations. Bulgarian biotechnology company Micar21 has discovered a new drug molecule for the treatment of COVID-19. Naicoms, which has developed a telemedicine platform, has announced that it is available free of charge. Checkpoint Cardio has announced that it has developed a device that allows telemonitoring and remote monitoring of patients with coronavirus symptoms [25].

\section{CONCLUSION}

During a pandemic telemedicine is crucial because patients should always have access to medical care. In order to discontinue the transmission of the dis- 
ease, especially among patients who are at increased risk of infection, health systems must rely on remote medical examinations and electronic prescriptions. Authorities must make efforts to lift, at least temporarily, restrictions on the use of telehealth, to help efforts to reduce patients' exposure to the coronavirus and to guarantee public health and safety.

Acknowledgements: Our article has not beeen funded. Each author has contributed with data collection, analyzes, writing and study design.

Disclosure summary: The authors have nothing to disclose.

\section{REFERENCES}

1. WHO. Coronavirus (COVID-19) outbreak. Available from: https://www.who.int/westernpacific/emergencies/covid-19

2. Pronovost PJ, Angus DC, Dorman T, et al. Physician staing patterns and clinical outcomes in critically ill patients: a systematic review. JAMA, 2002, 288(17), 2151-2162.

3. Latifi R, Merrell RC, Doarn CR, et al. „Initiate-build-operatetransfer"- a strategy for establishing sustainable telemedicine programs in developing countries: initial lessons from the Balkans. Telemedicine and e-Health, 2009, 15(14), 956-969.

4. Martínez A, Villarroel V, Seoane J, et al. Analysis of information and communication needs in rural primary health care in developing countries. IEEE Transactions on Information Technology in Biomedicine, 2005, 9(1), 66-72.

5. Pradhan MR. ICTs application for better health in Nepal. Kathmandu University Medical Journal, 2004, 2(2), 157-163.

6. Wootton R. Telemedicine support for the developing world. Journal of Telemedicine and Telecare, 2008, 14(3), 109-114.

7. Kaplan WA. Can the ubiquitous power of mobile phones be used to improve health outcomes in developing countries? Globalization and Health, 2006, 23(2), 9.

8. Rockwell KR, Gilroy AS. Incorporating Telemedicine as Part of COVID-19 Outbreak Response Systems. The American Journal of Managed Care, 2020 March 19. Available from: https://www.ajmc.com/journals/issue/2020/2020-vol26-n4/ incorporating-telemedicine-as-part-of-covid19-outbreak-response-systems

9. Coronavirus Preparedness and Response Supplemental Appropriation Act of 2020, HR 6074, 116th Cong, 2nd Sess, 2020.

10. Coding for Phone Calls, Internet and Telehealth Consultations. American Academy of Ophthalmology. 2020 May 5. Available from: https://www.aao.org/practice-management/ news-detail/coding-phone-calls-internet-telehealth-consult.

11. TMB responding to Gov. Abbott's state disaster declaration [news release]. Austin, TX: Texas Medical Board; 2020 March 14. Available from: tmb.state.tx.us/dl/920E0677-1BAF-C306781B-A570AD6795A1.

12. Moga-Paler $M$, Surugiu $A$, Sirakova $I$, et al. European Union: e-Healthcare and Telehealth Become Vital to Tackling COVID-19 in CEE. Schoenherr Attorneys at Law.
2020 March 25. Available from: https://www.mondaq.com/ Austria/Food-Drugs-Healthcare-Life-Sciences/907550/EHealthcare-And-Telehealth-Become-Vital-To-Tackling-COVID-19-In-CEE.

13. Telemedicine services multiply in Romania amid Covid-19. 2020 April 13. Available from: https://www.romania-insider. com/telemedicine-services-ro-apr-2020.

14. Ordinance №3 of 6. Oct. 2017 for approval of the Medical Standard "Emergency Medicine", section 3, item 5 Available from: https://www.mh.government.bg/media/filer_public/2018/04/10/naredba_3_ot_6102017_g_za_utvyrjdavane_ na_medicinski_standart_spesna_medicina.pdf.

15. Ordinance № 16 of 30 July 2014 on the terms and conditions for registration of rare diseases and on the expert centers and reference networks for rare diseases, Article 23, paragraph (2) Available from: http://ncpha.government.bg/files/komisia_ rare_diseases/Naredba_16_MH.pdf.

16. Telemedicine. Tools for Diagnostics, Triage. Available from: https://www.digital.health/covid-19.

17. Tyto Care. Available from: https://www.tytocare.com/cmp/ covid-19/\#form_section.

18. Siwicki B. Telemedicine during COVID-19: Benefits, limitations, burdens, adaptation. HealthcarelTNews, 2020 March 19 Available from: https://www.healthcareitnews.com/news/ telemedicine-during-covid-19-benefits-limitations-burdensadaptation.

19. mHealth solutions for managing the COVID-19 outbreak. Available from: http://mhealth-hub.org/mhealth-solutionsagainst-covid-19.

20. Brodwin E. Telehealth can help fight the novel coronavirus, but U.S. challenges could limit its potential. Statnews, 2020 February 28. Available from: https://www.statnews. com/2020/02/28/coronavirus-telehealth-digital-health-ushospitals-companies-face-challenges/.

21. Muoio D. Apple's Siri now walks worried users through their COVID-19 symptoms. Mobihealth news, 2020 March 24. Available from: https://www.mobihealthnews.com/news/ apples-siri-now-walks-worried-users-through-their-covid19-symptoms.

22. Lovett L. AMA launches Telemedicine Quick Reference Guide to help docs implement tech during COVID-19. Mobihealth news, 2020 March 25, Available from: https://www.mobihealthnews.com/news/ama-launches-telemedicine-quickreference-guide-help-docs-implement-tech-during-covid19?utm_source=dlvr.it\&utm_medium=facebook.

23. Using Telehealth to Care for Patients During the COVID-19 Pandemic. American Academy of Family Physicians. 2020 May 9. Available from: https://www.aafp.org/patient-care/ emergency/2019-coronavirus/telehealth.html.

24. O'Neill PH. Apple and Google are building coronavirus tracking into iOS and Android. MIT Technology Review, 2020 April 10. Available from: https://www.technologyreview. com/2020/04/10/999213/apple-and-google-are-buildingcoronavirus-tracking-into-ios-and-android/

25. The European Commission: Startups With Solutions To Tackle The COVID-19 Outbreak Should Apply For EIC Funding Before Wednesday. Trending Topics, 2020 March 16. Available from: https://www.trendingtopics.at/bulgaria/the-european-commission-startups-with-solutions-to-tackle-the-covid19-outbreak-should-apply-for-eic-funding-before-wednesday/

Received: 08 June 2020, Accepted: 26 July 2020 